\title{
Short Report: Novel non-contiguous exon duplication in choroideremia
}

Authors: Thomas L Edwards ${ }^{1,2^{*}}$, Jonathan Williams ${ }^{35^{*}}$, Maria I Patrício ${ }^{1}$, Matthew

P Simunovic ${ }^{1,2}$, Morag Shanks ${ }^{3}$, Penny Clouston $^{3}$, Robert E MacLaren ${ }^{1,2,4}$

1) Nuffield Laboratory of Ophthalmology, Department of Clinical Neurosciences, University of Oxford, Oxford, United Kingdom

2) Oxford Eye Hospital, Oxford University Hospital NHS Foundation Trust, Oxford, United Kingdom

3) Oxford Medical Genetics Laboratories, Churchill Hospital, Oxford University Hospital NHS Foundation Trust, Oxford United Kingdom

4) Moorfields Eye Hospital-UCL Institute of Ophthalmology NIHR Biomedical Research Centre, London, United Kingdom

${ }^{\S}$ Corresponding author. Oxford Medical Genetics Laboratories, Churchill Hospital, Old Road, Headington, Oxford, OX3 7LE, United Kingdom. Phone:

01865 225290. Email address: jonathan.williams2@ouh.nhs.uk

"These authors contributed equally to this work.

Word count: 2460

\section{Acknowledgements}

This is the author manuscript accepted for publication and has undergone full peer review but has not been through the copyediting, typesetting, pagination and proofreading process, which may lead to differences between this version and the Version of Record. Please cite this article as doi: $10.1111 /$ cge.13021

This article is protected by copyright. All rights reserved. 
This work was funded in part by a grant to TE from the Nuffield Trust. This work was also funded by the Oxford University Hospitals Foundation Trust NIHR Biomedical Research Centre and Fight for Sight.

\section{Conflict of Interest}

The authors declare no relevant conflict of interest

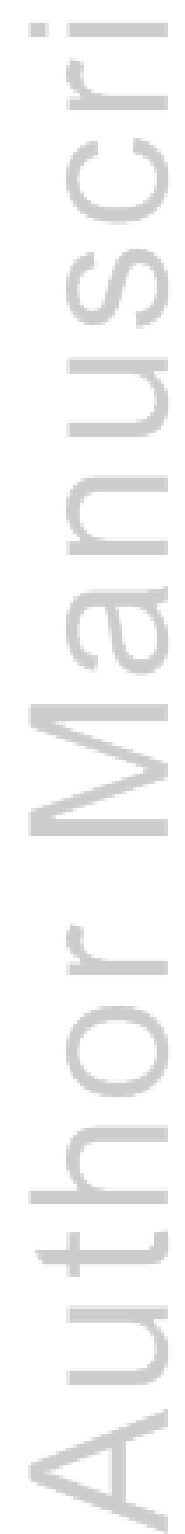

This article is protected by copyright. All rights reserved. 


\section{Abstract}

The importance of establishing a genetic diagnosis in patients with a choroideremia phenotype has been underscored by the advent of gene replacement therapy for this condition. Here we describe a complex imbalance at the CHM locus in a male patient with classical disease. At the DNA level, this imbalance consists of two non-contiguous duplications (exons 1-2, and 9-12). Further characterization suggests the generation of two independent $\mathrm{CHM}$ transcriptional units, one of which may produce a deleted form of the REP1 protein. Expression of such a type of aberrant protein in photoreceptors may have important implications when considering gene therapy for this disorder.

Key Words- Retinal Dystrophy, Ophthalmology, Choroideremia, Genetic Testing, Structural Variation, Copy Number Variation

\section{Introduction}

Choroideremia (OMIM phenotype reference: 303100 ) is an X-linked retinal degeneration that results in progressive concentric field loss during early adulthood, before involving central vision typically by the fourth decade ${ }^{1}$. It is due to mutations in CHM, on chromosome Xq21 (OMIM gene reference: 300390), that lead to deficiency of the Rab escort protein 1(REP1), causing under-prenylation of Rab GTPases, essential for membrane trafficking and vesicle transport in photoreceptors ${ }^{2}$. 
The majority of disease-associated mutations in CHM result in a loss of detectable REP1 and include whole and partial deletions gene deletions, and single nucleotide variants leading to premature termination of protein translation through a variety of mechanisms ${ }^{3-6}$. Mutations maintaining the $\mathrm{CHM}$ reading frame are rare; however, missense variants predicted to result in decreased protein stability, as well as variants leading to in-frame exon skipping have also been reported ${ }^{3,5}$.

Here we present a novel $C H M$ variant identified in a male patient with typical choroideremia consisting of two non-contiguous duplications that has been further characterized at the RNA level by Reverse Transcription PCR (RTPCR).

\section{Patients and Methods}

The patient is a 44 year-old male with a history of 'retinitis pigmentosa' (RP) who first noticed a decline in night vision during late childhood. He had no associated systemic features and there was no known family history of eye disorders. The best-corrected visual acuity was $6 / 24$ and 6/15 in the right and left eye respectively. Fundoscopy (Spectralis, Heidelberg Engineering,

Germany) showed extensive symmetrical chorioretinal atrophy with sparing of the posterior poles over an area corresponding to a well-demarcated island of residual fundus autofluorescence (Figure 1). Spectral domain optical coherence tomography (Spectralis, Heidelberg Engineering, Germany) revealed 
widespread chorioretinal thinning in those regions outside the island of autofluorescence. A topographical map of macular threshold sensitivity was performed by microperimetry testing (Maia, CenterVue, Italy), which showed relatively sustained retinal function over the island of autofluorescence, which was extinguished precipitously outside its border.

The coding regions of 55 genes associated with RP were sequenced in DNA isolated from venous blood, using a custom design Haloplex enrichment kit (Agilent Technologies) on the llumina MiSeq platform. Data analysis was performed using an in-house pipeline.

Dosage analysis of $\mathrm{CHM}$ was performed using MLPA (Probe mix P366, MRCHolland, Amsterdam, Netherlands) with data analysis performed in Coffalyser (MRC-Holland). REP1 protein expression was assayed using immunoblot on lysed primary fibroblasts obtained from patients' skin biopsies (UK REC reference 15/WA/0087) probed with an anti-REP1 monoclonal antibody (EMD Millipore, Hertfordshire, UK) with $\beta$ actin used as a loading control.

For RT-PCR analysis, total RNA from whole blood was extracted using the QIAamp RNA Blood Mini Kit (Qiagen, Manchester, UK). cDNA synthesis was performed using random primed synthesis with SuperScript III (Life Technologies, Paisley, UK) . cDNA libraries were amplified using KAPA 2G Fast polymerase (Kapa Biosystems, Cape Town, South Africa) prior to Sanger 
sequencing on an $\mathrm{ABI} 3730$ using BigDye V3.1 chemistry (Life Technologies, Netherlands).

Variants are described based on the RefSeq CHM transcript NM_000390.3. Work carried out in this study has received ethics approval from the London West London \& GTAC research ethics committee (reference 15/LO/1379) as part of the REGENERATE study (ClinicalTrials.gov ref NCT02407678).

\section{Results}

Next Generation Sequencing (NGS) of 55 genes associated with RP did not detect a pathological sequence variant in our patient; however, read depth analysis was suggestive of a putative complex rearrangement in $\mathrm{CHM}$ which was confirmed using Multiplex Ligation-dependent Probe Amplification (MLPA, Figure 2A). The relative copy number of exons $1-2$ and exons 9-12 were doubled, indicating the presence of two non-contiguous CHM exon duplications.

An identical MLPA result was observed when analyzing DNA extracted from the patient's mother, consistent with X-linked inheritance and her carrier status. However, because MLPA is unable to provide positional information with regards to the insertion point of duplicated sequence, there remained the possibility of an intact $\mathrm{CHM}$ gene with the duplicated material inserted elsewhere in the genome in both the patient and his mother. 
To explore this, an assay of REP1 using immunoblot, was performed (Figure 2B). An absence of REP1 in cultured skin fibroblasts was confirmed, consistent with pathogenicity of the non-contiguous duplication, and a clinical diagnosis of choroidermia. Efficacy of the assay was demonstrated by detection of REP1 in two positive control (non-choroideremia) cell lines.

As immunoblot analysis did not detect any REP1 signal in our patient, we next sought to fully characterize the molecular nature of the non-contiguous duplication. We chose an approach based on analysis of CHM RNA using RTPCR. cDNA libraries were initially amplified using seven different primer sets designed across splice sites throughout the reference CHM transcript (NM_000390.3, results not shown). In this initial analysis, a primer pair amplifying between exons 8 and 13 failed to generate a product from the patient's library, indicating that the non-contiguous duplication was affecting normal transcript production between these sites.

A second set of PCRs was carried out using three different primer sets, one amplifying between exons 1 and 12, a second between exons 1 and 15, and a final set between exons 1 and 13. As shown in Figure 3A, the exon 1-12 primer set produced a predominant product in the control sample consistent with the normal pattern of CHM splicing. When using the patient's cDNA library as template, a band matching that in the control and a second product of approximately 475 bp was generated. Using both the exon 1-15 and exon 1-13 
primer sets a single product corresponding to the normal $\mathrm{CHM}$ transcript was amplified from the control library, while two main products of significantly smaller size were amplified from the patient library confirming loss of normal transcript expression.

To better characterize the smaller products from the patient sample, purified products from the exon 1-15 primer set (identified in Figure 3A by asterisks) were sequenced. Figure 3B shows sequence analysis of the products from the patient's library, revealing the predominant product ( ${ }^{*} 2$ in Figure $3 \mathrm{~A}$ ) to be composed of exons 1-2 spliced directly onto exons 9-15; analysis of the product from the control library ( ${ }^{*} 1$ in Figure $3 A$ ) confirmed normal splicing of exons 2-3 (see Figure $3 \mathrm{C})$. The smaller, less intense, product from the patient sample $\left({ }^{*} 3\right.$ in Figure 3A) appeared to be generated through an alternate splicing event between exons 9 and 12 .

\section{Discussion}

In this case, we have characterized a novel, pathogenic, non-contiguous duplication in $C H M$ comprising exons 1-2 and exons 9-12. Based on the results of cDNA analysis in this patient, we propose the non-contiguous duplication consists of two CHM transcriptional units (see Figure 3D). The first composed of exons 1 to 12 (Unit $A$ ), and the second containing the duplicated exons $(1,2,9$, 10, 11, and 12) along with single copy exons 13-15 (Unit B). Using HGVS 
nomenclature, this variant is best described at the RNA level as two transcripts originating from a single chromosome: r.[1511_*3450del;117_1166del].

Given that Unit A is predicted to lack the transcription termination and polyadenylation signals of wild type CHM it is unclear whether it will produce functional mRNA transcripts. Conversely, the presence of both the proximal and terminal segments of the $\mathrm{CHM}$ gene in Unit $\mathrm{B}$ is consistent with the production of functional mRNA capable of protein translation; the predominant transcript from this unit would be predicted to result in a REP1 protein with an in-frame deletion of 350 amino acids from Arg40 to Arg389 (HGVS:

p.(Arg40_Arg389del)). Such putative protein was also predicted to be translated in a choroideremia patient with a simple deletion of $\mathrm{CHM}$ exons 3-8 ${ }^{7}$, and may evade detection by our anti-REP1 monoclonal antibody, which was raised against the C-terminus of recombinant human REP1( i.e. exons 6-15 inclusive). One explanation for how this rearrangement may have occurred is displayed in Figure 3E. Misalignment and subsequent recombination between a normal $\mathrm{X}$ chromosome and one containing a $C H M$ gene with a deletion of exons 3-8, as also reported in a family by McTaggart et al, may have generated this complex locus ${ }^{7}$.<smiles>[CH]1CCC1</smiles>

Production of a deleted form of REP1 could have implications on gene therapy. To-date, clinical trials have been performed on a null background with respect 
to REP1 expression ${ }^{8,9}$. Indeed, complete loss of protein in the majority of patients has been highlighted as an advantage in determining dosage levels in clinical trials as compared to disorders such as RPE65-associated Leber Congenital Amaurosis (LCA) ${ }^{9,10}$.

Detailed assessment of choroideremia patients with missense variants versus those with absent REP1 is not suggestive of phenotypic differences in these cohorts $^{4,6}$. However, the impact of residual mutant REP1 expression on clinical outcome in the context of gene therapy remains to be assessed, with suggestions that interference could impact success rates ${ }^{6}$. Furthermore, it is interesting to note that misfolding of mutant RPE65 has been shown to lead to cytotoxic aggregates in the photoreceptors of LCA patients: an alternate pathogenic mechanism that cannot be treated through gene replacement therapy that should also be explored in rare cases of choroideremia maintaining mutant REP1 expression ${ }^{11}$.

To the best of our knowledge, this represents the first complex rearrangement to be described in $\mathrm{CHM}$, and highlights the utility of supplementing NGS with further genetic testing when there is a high index of clinical suspicion.

\section{References}

1. Heon E, Alabduljalil T, McGuigan IIIDB, et al. Visual Function and Central Retinal Structure in ChoroideremiaNatural History of 
Choroideremia. Investigative Ophthalmology \& Visual Science. 2016;57(9):ОСТ377-ОСТ387.

2. Seabra M, Brown M, Goldstein J. Retinal degeneration in choroideremia: deficiency of rab geranylgeranyl transferase. Science. 1993;259(5093):377-381.

3.= Esposito G, De Falco F, Tinto N, et al. Comprehensive mutation analysis (20 families) of the choroideremia gene reveals a missense variant that prevents the binding of REP1 with rab geranylgeranyl transferase. Human Mutation. 2011;32(12):1460-1469.

4. Freund PR, Sergeev YV, MacDonald IM. Analysis of a large choroideremia dataset does not suggest a preference for inclusion of certain genotypes in future trials of gene therapy. Molecular Genetics \& Genomic Medicine. 2016;4(3):344-358.

5. J dufld-

This article is protected by copyright. All rights reserved. 
7. McTaggart KE, Tran M, Mah DY, Lai SW, Nesslinger NJ, MacDonald IM. Mutational analysis of patients with the diagnosis of choroideremia. Human Mutation. 2002;20(3):189-196.

8. Edwards TL, Jolly JK, Groppe M, et al. Visual Acuity after Retinal Gene Therapy for Choroideremia. New England Journal of Medicine.

$=2016 ; 374(20): 1996-1998$.

9. MacLaren RE, Groppe M, Barnard AR, et al. Retinal gene therapy in patients with choroideremia: initial findings from a phase 1/2 clinical trial. The Lancet. 2014;383(9923):1129-1137.

10. Bainbridge JWB, Mehat MS, Sundaram V, et al. Long-Term Effect of Gene Therapy on Leber's Congenital Amaurosis. New England Journal of Medicine. 2015;372(20):1887-1897.

11. Li S, Izumi T, Hu J, et al. Rescue of Enzymatic Function for Diseaseassociated RPE65 Proteins Containing Various Missense Mutations in Non-active Sites. Journal of Biological Chemistry. July 4, 2014 2014;289(27):18943-18956. 


\section{Titles and Legends to Figures}

\section{Figure 1. Clinical phenotype}

(A) Colour fundus photographs; (B) autofluorescence images (Spectralis, Heidelberg Engineering, Germany); (C) microperimetry (Maia, CenterVue, Italy) and; (D) optical coherence tomography scans (Spectralis, Heidelberg Engineering, Germany).

\section{Figure 2. Multiplex Ligation-dependent Probe Amplification and REP1} expression.

(A) The relative copy number of exons 1-2 and exons 9-12 were doubled, indicating the presence of two non-contiguous exon duplications. (B) Immunoblot demonstrating absence of REP1 expression in the patient's skin fibroblasts. A band of the correct size $(83 \mathrm{kDa})$ was detected in the fibroblasts of 2 other male patients with no mutation in $\mathrm{CHM}$ (Control \#1 and \#2). $\beta$ actin was used as a loading control. Key: $\mathrm{CHM}=$ patient sample; Control \#1 and \#2 = control samples from unaffected males \#1 and \#2.

Figure 3. Proposed organization of the transcription units and a model for their generation based on PCR and Sanger sequencing analysis (A) PCR analysis using primers amplifying between exons 1-12, exons 1-15 and exons 1-13 for the wild-type (control) and mutant (patient) CHM cDNA library as 
a template. Products marked with asterisks were further purified prior to Sanger sequencing. (B) The top chromatogram, generated from the larger of the two patient fragments ( ${ }^{*} 2$ in Panel A), demonstrates splicing between exons 2 and 9 generating a transcript consisting of exons 1-2 spliced onto exons 9-15. The lower chromatogram highlights splicing between exons 9 and 12 observed in the smaller of the two products resulting in an alternative transcript lacking exons 10 and 11 ( ${ }^{*} 3$ in Panel A). (C) A portion of sequencing data from the product generated by PCR using primers specific to exons 1 and 15 and using the control cDNA library as a template demonstrating the expected pattern of splicing between exons 2 and 3 ( ${ }^{*} 1$ in Panel A). (D) We propose that the CHM locus in our patient consists of two individual transcription units (A and $B)$. (E) We propose a model where misalignment and subsequent recombination between a normal $\mathrm{X}$ chromosome and one containing a CHM gene with a deletion of exons 3-8 results in the generation of this complex locus.

This article is protected by copyright. All rights reserved. 


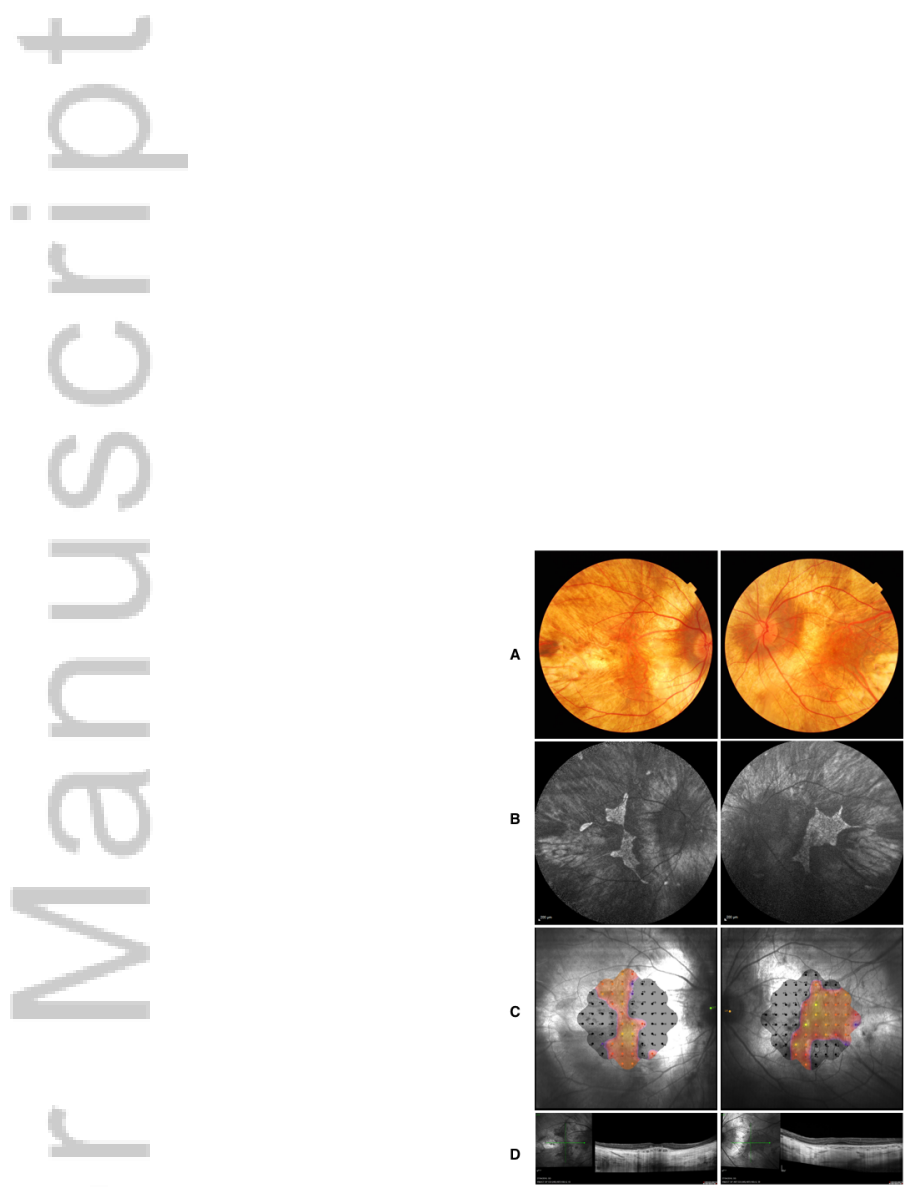

Figure_1_300.tiff

This article is protected by copyright. All rights reserved. 


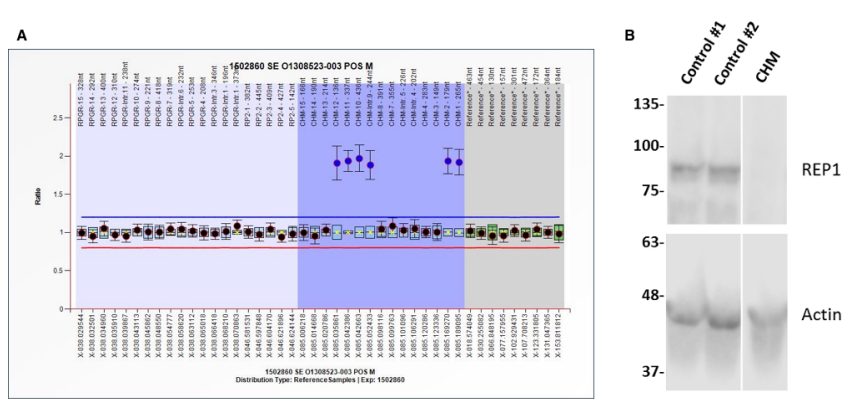

Figure_2_revised_300.tiff

This article is protected by copyright. All rights reserved. 


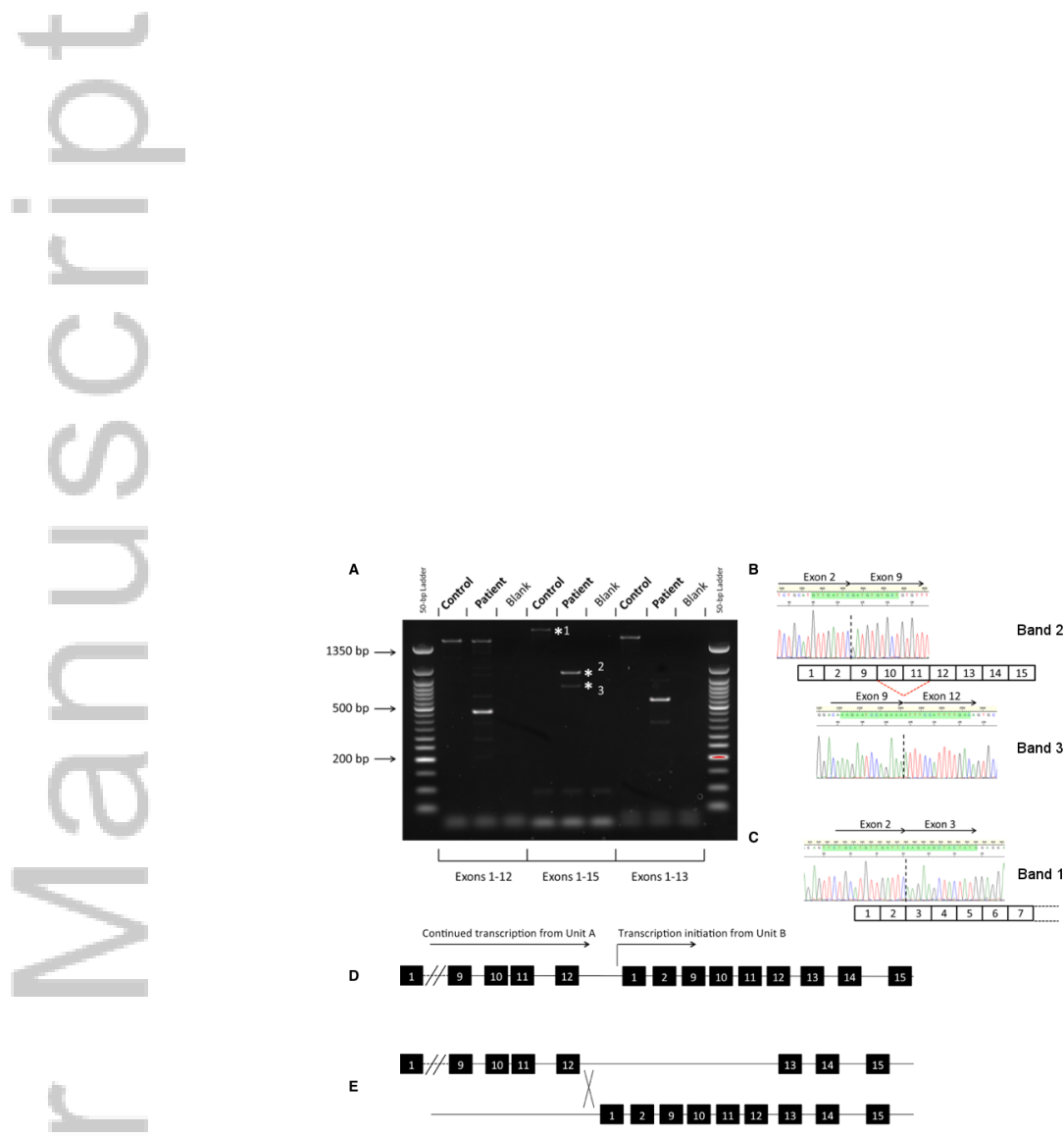

Figure_3_revised_300.tiff

This article is protected by copyright. All rights reserved. 

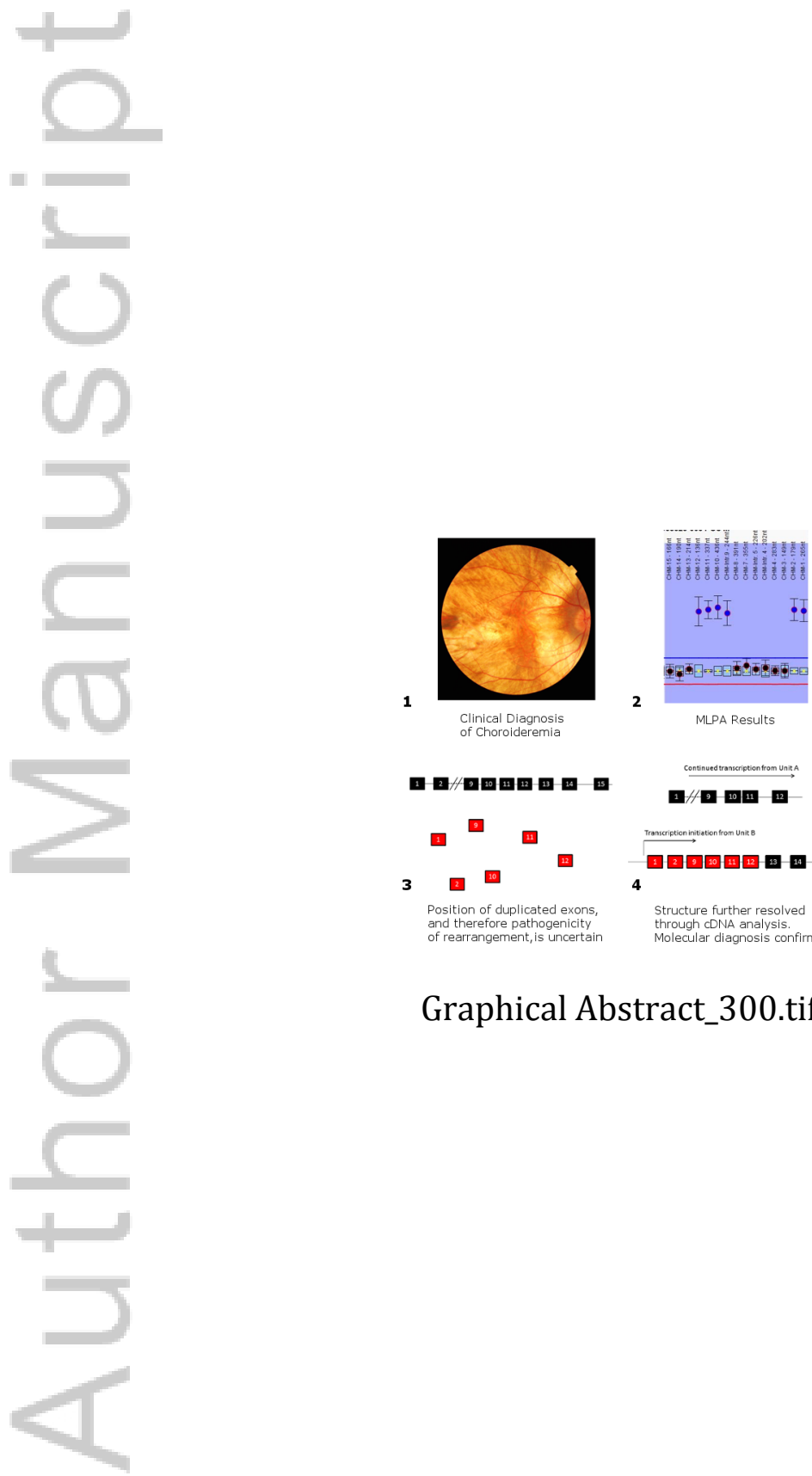

1/9 80110

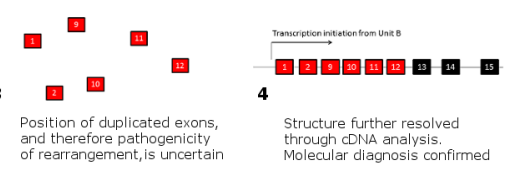

Graphical Abstract_300.tif

This article is protected by copyright. All rights reserved. 


\section{University Library}

\section{- M M N E R VA A gateway to Melbourne's research publications}

Minerva Access is the Institutional Repository of The University of Melbourne

\section{Author/s:}

Edwards, TL;Williams, J;Patricio, Ml;Simunovic, MP;Shanks, M;Clouston, P;MacLaren, RE

Title:

Novel non-contiguous exon duplication in choroideremia

Date:

2018-01-01

Citation:

Edwards, T. L., Williams, J., Patricio, M. I., Simunovic, M. P., Shanks, M., Clouston, P. \& MacLaren, R. E. (2018). Novel non-contiguous exon duplication in choroideremia. CLINICAL GENETICS, 93 (1), pp.144-148. https://doi.org/10.1111/cge.13021.

Persistent Link:

http://hdl.handle.net/11343/292782 